\title{
Phase-lag heat conduction: decay rates for limit problems and well-posedness
}

\author{
Karin Borgmeyer, Ramón Quintanilla ${ }^{1}$ and Reinhard Racke
}

\begin{abstract}
In two recent papers the authors have studied conditions on the relaxation parameters in order to guarantee the stability or instability of solutions for the Taylor approximations to dual-phase-lag and three-phase-lag heat conduction equations. However, for several limit cases relating to the parameters the kind of stability was unclear. Here we analyze these limit cases and clarify whether we can expect exponential or slow decay for the solutions. Moreover, rather general well-posedness results for three-phase-lag models are presented. Finally, the exponential stability expected by spectral analysis is rigorously proved exemplarily.
\end{abstract}

\section{Introduction}

It is well known that Fourier's heat conduction theory implies that the thermal disturbance at some point in a solid will be felt instantly anywhere, however distant. From a physical point of view this is a drawback of the model because it predicts that heat waves propagate with infinite speed. To save the principle of causality, several heat conduction theories were suggested in the second part of the last century (see $[2,6,7]$ ). In the books $[10,20$, 22 ], several studies concerning the applicability of nonclassical thermoelastic theories are considered.

In 1995 Tzou [21] suggested a modification of Fourier's law. He proposed a theory of a thermal flux with delay. It is based on the constitutive equation

$$
q\left(x, t+\tau_{q}\right)=-k \nabla T\left(x, t+\tau_{T}\right), \quad k>0 .
$$

Here $q$ is the heat flux vector and $T$ is the temperature. This equation proposes that the temperature gradient established across a material volume at position $x$ at time $t+\tau_{T}$ results in a heat flux to flow at a different instant of time $t+\tau_{q}$. This delay should be understood in terms of the microstructure of the material. Recently, Choudhuri [19] proposed an extension of Tzou's proposition. The constitutive equation for the heat flux

\footnotetext{
${ }^{0}$ AMS subject classification: 35 L 35, 80 A 20

Keywords and phrases: hyperbolic models in heat conduction, stability

${ }^{1}$ Supported by the project "Ecuaciones en Derivadas Parciales en Termomecánica. Teoría y Aplicaciones" (MTM2009-08150) of the Spanish Ministry of Science and Technology)
} 
vector is

$$
q\left(x, t+\tau_{q}\right)=-\left(k \nabla T\left(x, t+\tau_{T}\right)+k^{*} \nabla \nu\left(x, t+\tau_{\nu}\right)\right) .
$$

Here $\nu$ is the thermal displacement that satisfies $\dot{\nu}=T$. It seems that the aim of Choudhuri was to establish a mathematical model based on delay in such a way that the Taylor approximations recover the models of Green and Naghdi [4, 5].

Both theories are strongly based on an intuitive point of view, but there is no a priori thermomechanical foundation. Unfortunately, it can be proved that, when we adjoin these constitutive equations with the classical energy equation

$$
-\operatorname{div} q(x, t)=c \dot{T}(x, t), \quad c>0,
$$

there always exists a sequence of solutions $T_{n}(t, x)=\exp \left(\omega_{n} t\right) \Phi_{n}(x)$ such that the real part Re $\omega_{n}$ tends to infinity [3]. This result implies that the associated mathematical problem is ill posed in the sense of Hadamard. Of course, this is not suitable for a heat conduction theory and disagrees with what one would expect a priori. For this reason a big interest has been developed to study different Taylor approximations to these equations $[1,8,11,12,13,14,15,16,17,18]$. These alternative theories allow to obtain the stability of solutions and the well-posedness of the problems. In particular, the contributions $[16,18]$ gave conditions to guarantee stability or instability of solutions for the different theories. However, we should mention that few attention has been dedicated to the limit cases for the parameters. That is, we proved that when a strict inequality holds stability or instability can be obtained. However, we have not yet paid attention to what happens in the more difficult case of equalities. This is one main object of the present contribution. We wish to see if the decay of solutions is fast (exponential) or only slow (i.e. not exponential) in these limit cases. It is worthwhile mentioning that our contribution is also motivated by the paper of Ignaczak [9] who proved that, in some limit cases, progressive heat waves behave differently. Our analysis is devoted to clarify when the different theories should be accepted or rejected.

Assuming w.l.o.g. $c=1$ in (1.3) in the sequel, two of the equations proposed by Tzou, obtained by means of a formal Taylor approximation to the delay equation (1.1) and leading to dual-phase-lag equations, are

$$
\dot{T}+\tau_{q} \ddot{T}+\frac{\tau_{q}^{2}}{2} \dddot{T}=k \Delta T+k \tau_{T} \triangle \dot{T}
$$

and

$$
\dot{T}+\tau_{q} \ddot{T}+\frac{\tau_{q}^{2}}{2} \dddot{T}=k \triangle T+k \tau_{T} \triangle \dot{T}+k \frac{\tau_{T}^{2}}{2} \triangle \ddot{T} .
$$

(i) Equation (1.4) is hyperbolic. We recall that we proved that when $\tau_{q}<2 \tau_{T}$ the problem is exponentially stable, whereas, when $\tau_{q}>2 \tau_{T}$, it is unstable (see [16]). When 
$\tau_{q}=2 \tau_{T}$ the problem will also be stable, but we need to clarify whether the decay is of exponential type. In fact, we will prove that the answer is negative and that there is slow decay.

(ii) Equation (1.5) is more difficult to study. In fact, the best that we know is that the point spectrum is far away from the imaginary axis when $\tau_{T}>(2-\sqrt{3}) \tau_{q}$ (see [16]). We wish to analyze what happens in the limit case $\tau_{T}=(2-\sqrt{3}) \tau_{q}$.

Using formal Taylor approximations to the delay equation (1.2), Choudhuri proposed the following couple of equations, representing three-phase-lag problems,

$$
\ddot{T}+\tau_{q} \dddot{T}=k^{*} \triangle T+\tau_{\nu}^{*} \triangle \dot{T}+k \tau_{T} \triangle \ddot{T}
$$

and

$$
\ddot{T}+\tau_{q} \dddot{T}+\frac{\tau_{q}^{2}}{2} \dddot{T}=k^{*} \triangle T+\tau_{\nu}^{*} \Delta \dot{T}+k \tau_{T} \Delta \ddot{T}
$$

(iii) We recall that equation (1.6) is exponentially stable if $\tau_{\nu}^{*}>k^{*} \tau_{q}$, where $\tau_{\nu}^{*}$ is defined by $k^{*} \tau_{\nu}+k$ (see [18]), and that, depending on the domain, it can be unstable if $\tau_{\nu}^{*}<k^{*} \tau_{q}$. Here we want to clarify the case $\tau_{\nu}^{*}=k^{*} \tau_{q}$. We will see that the point spectrum is at a positive distance to the imaginary axis which suggests exponential stability, that will be proved using energy methods.

(iv) Equation (1.7) is exponentially stable when $k^{*} \tau_{q}<\tau_{\nu}^{*}<\frac{2 k \tau_{T}}{\tau_{q}}$ (see [18]). We here analyze several limit cases.

For the limit case $\tau_{\nu}^{*}=k^{*} \tau_{q}$ in (iii) and in (iv), where exponential stability will be suggested by the spectral analysis, we will demonstrate this exponential stability analytically using energy methods.

Moreover, we give a rigorous and rather general analysis of the well-posedness of (three-)phase-lag inital-boundary value problems.

The paper is organized as follows: In sections 2-5 we analyze the limit cases with respect to exponential or slow decay by means of spectral analysis. In section 6 we discuss the wellposedness of (three-)phase-lag problems in a general setting following [1]. Exponential stability for the limit case $\tau_{\nu}^{*}=k^{*} \tau_{q}$ is proved in section 7 for (iii), and in section 8 for (iv).

\section{Spectral analysis for the limit case of (1.4)}

In this section we analyze equation (1.4) when $\tau_{q}=2 \tau_{T}$. Then the equation turns into

$$
\dot{T}+2 \tau \ddot{T}+2 \tau^{2} \dddot{T}=k \triangle T+k \tau \triangle \dot{T}
$$

where $\tau=\tau_{T}$, dropping the sub-index to simplify the expressions. Let us consider functions of the form

$$
T(x, t)=\exp (\omega t) \Phi_{n}(x)
$$


where $\Phi_{n}$ satisfies the eigenvalue problem

$$
\triangle \Phi_{n}+\lambda_{n} \Phi_{n}=0 \quad \text { in } \Omega, \quad \Phi_{n}=0 \quad \text { on } \partial \Omega .
$$

Thus, $\left(\lambda_{n}\right)_{n}$ are the positive eigenvalues of the usual Laplace operator in $\Omega$, where $\Omega$ represents the body as a bounded domain in $\mathbb{R}^{d}, d \geq 1$, and is assumed to have a smooth boundary $\partial \Omega$.

The function in (2.2) is a solution of (1.4) whenever $\omega$ satisfies the polynomial equation

$$
2 \tau^{2} \omega^{3}+2 \tau \omega^{2}+\left(1+k \tau \lambda_{n}\right) \omega+k \lambda_{n}=0
$$

The zeros of this equation correspond to the spectrum of that operator $\mathcal{A}$ which would arise if we transformed the equation to a first-order system of the type $V_{t}+\mathcal{A} V=0$.

We would like to see if the solutions are near to the imaginary axis. We will show that for every positive $\epsilon$, there always exist elements of the point spectrum on the right of the line $\operatorname{Re}\{\omega\}=-\epsilon$. For this reason we introduce $z:=\omega+\epsilon$, i.e. we exchange $\omega$ by $z-\epsilon$, and we obtain the equation

$$
z^{3}+l_{1} z^{2}+l_{2} z+l_{3}=0
$$

where

$$
l_{1}=\tau^{-1}-3 \epsilon, l_{2}=\frac{1+k \tau \lambda_{n}}{2 \tau^{2}}+3 \epsilon^{2}-\frac{2 \epsilon}{\tau}, l_{3}=\frac{k \lambda_{n}}{2 \tau^{2}}-\epsilon^{3}+\frac{\epsilon^{2}}{\tau}-\frac{\left(1+k \tau \lambda_{n}\right) \epsilon}{2 \tau^{2}} .
$$

To prove that there exist solutions to equation (2.3) which are on the right of the line $\operatorname{Re}\{\omega\}=-\epsilon$, we will show that there are solutions to (2.4) with positive real part. By the Hurwitz criterion, all three roots of the equation have negative real parts if and only if the following inequalities

$$
l_{j}>0, j=1,2,3, \quad l_{1} l_{2}>l_{3},
$$

hold. In our case we can always take $\epsilon$ small enough to guarantee $l_{i}>0$ for every $i=1,2,3$. However, a direct calculation shows that

$$
l_{1} l_{2}-l_{3}=\frac{1}{2 \tau^{3}}-2 \epsilon\left(\frac{1+k \tau \lambda_{n}}{2 \tau^{2}}\right)-\frac{2 \epsilon}{\tau^{2}}+\epsilon^{2} \Psi,
$$

where $\Psi$ is bounded because it can be obtained as an expression which depends on the parameter and $\epsilon$, but which does not depend on $\lambda_{n}$. As $\lambda_{n}$ growths and we select $\epsilon$ as small as we want (but positive), we can always select $n$ such that the right-hand side of (2.6) is less than zero. Thus, we conclude that in this case we can always find elements of the point spectrum on the right of the line $R e\{\omega\}=-\epsilon$. Therefore, we do not have exponential decay of the solutions.

Theorem 2.1. In the limit case of (1.4) we have slow decay. 


\section{Spectral analysis for the limit case of (1.5)}

It was proved that when $\tau_{T}>(2-\sqrt{3}) \tau_{q}$ the equation is exponentially stable. We here consider the limit case when $\tau_{T}=(2-\sqrt{3}) \tau_{q}$. Equation (1.5) can be written as

$$
\dot{T}+\tau \ddot{T}+\frac{\tau^{2}}{2} \dddot{T}=k \Delta T+k(2-\sqrt{3}) \tau \Delta \dot{T}+k \frac{(2-\sqrt{3})^{2} \tau^{2}}{2} \triangle \ddot{T},
$$

where $\tau=\tau_{q}$.

The functions of the form (2.2) are solutions whenever $\omega$ satisfies the polynomial equation

$$
\frac{\tau^{2}}{2} \omega^{3}+\tau\left(1+\frac{(2-\sqrt{3})^{2} \tau k \lambda_{n}}{2}\right) \omega^{2}+\left(1+(2-\sqrt{3}) \tau k \lambda_{n}\right) \omega+k \lambda_{n}=0 .
$$

Again exchanging $\omega$ by $z-\epsilon$ we obtain the equation (2.4), now with

$$
\begin{gathered}
l_{1}=\tau^{-1}\left(2+(2-\sqrt{3})^{2} \tau k \lambda_{n}\right)-3 \epsilon \\
l_{2}=\frac{2}{\tau^{2}}\left(\left(1+(2-\sqrt{3}) \tau k \lambda_{n}\right)+\frac{3}{2} \tau^{2} \epsilon^{2}-\tau\left(2+(2-\sqrt{3})^{2} \tau k \lambda_{n}\right) \epsilon\right) \\
l_{3}=\frac{2}{\tau^{2}}\left(k \lambda_{n}-\frac{\tau^{2} \epsilon^{3}}{2}+\tau\left(1+\frac{(2-\sqrt{3})^{2}}{2} \tau k \lambda_{n}\right) \epsilon^{2}-\left(1+(2-\sqrt{3}) \tau k \lambda_{n}\right) \epsilon\right) .
\end{gathered}
$$

We see that $l_{j}>0$ if $\epsilon$ is small enough, independent of $\lambda_{n}$, and

$$
\begin{gathered}
l_{1} l_{2}-l_{3}=A \lambda_{n}^{2}-B \lambda_{n}+C=\left(\sqrt{A} \lambda_{n}-\frac{B}{2 \sqrt{A}}\right)^{2}+\left(C-\frac{B^{2}}{4 A}\right) \\
=\left(\sqrt{A} \lambda_{n}-\frac{B}{2 \sqrt{A}}\right)^{2}+\frac{1}{4 A}\left(4 A C-B^{2}\right),
\end{gathered}
$$

where

$$
\begin{gathered}
A=k(2-\sqrt{3})^{2}\left(\frac{2 k(2-\sqrt{3})}{\tau}-2 k(2-\sqrt{3})^{2} \epsilon\right), \\
B=\left(\frac{2 k}{\tau^{2}}+k(2-\sqrt{3})^{2} \epsilon^{2}-\frac{2 k(2-\sqrt{3}) \epsilon}{\tau}\right)+\left(3 \epsilon-\frac{2}{\tau}\right)\left(\frac{2 k(2-\sqrt{3})}{\tau}-2 k(2-\sqrt{3})^{2} \epsilon\right) \\
+k(2-\sqrt{3})^{2}\left(\frac{4 \epsilon}{\tau}-\frac{2}{\tau^{2}}-3 \epsilon^{2}\right), \\
C=\left(\frac{2}{\tau}-3 \epsilon\right)\left(\frac{2}{\tau^{2}}-\frac{4 \epsilon}{\tau}+3 \epsilon^{2}\right)-\left(\frac{2 \epsilon^{2}}{\tau}-\frac{2 \epsilon}{\tau^{2}}-\epsilon^{3}\right) .
\end{gathered}
$$


Marking the dependence on the parameters $n$ and $\epsilon$, we rewrite (3.2) as

$$
\left(l_{1} l_{2}-l_{3}\right)(n, \epsilon)=A(\epsilon)(\underbrace{\left(\lambda_{n}-\frac{B(\epsilon)}{2 A(\epsilon)}\right)^{2}}_{=: S_{1}(n, \epsilon)}+\underbrace{\frac{1}{4 A^{2}(\epsilon)}\left(4 A(\epsilon) C(\epsilon)-B^{2}(\epsilon)\right)}_{=: S_{2}(\epsilon)})
$$

First case:

$$
\forall n \in \mathbb{N}: \quad \lambda_{n} \neq \frac{B(0)}{2 A(0)}=\frac{3 \sqrt{3}-5}{k \tau(26-15 \sqrt{3})} .
$$

Then we conclude, since $0<\lambda_{n} \rightarrow \infty$ :

$$
\exists c_{0}>0 \exists \epsilon_{1}>0 \forall 0<\epsilon \leq \epsilon_{1} \forall n: S_{1}(n, \varepsilon) \geq c_{0} .
$$

Since we shall see in the discussion of the following second case that $S_{2}(\epsilon)=O(\epsilon)$ always holds, we conclude

$$
\exists c_{1}>0 \exists \epsilon_{2}>0 \forall 0<\epsilon \leq \epsilon_{2} \forall n:\left(l_{1} l_{2}-l_{3}\right)(n, \epsilon) \geq c_{1},
$$

which suggests the expected exponential decay in this case.

Second case:

$$
\exists n_{0} \in \mathbb{N}: \lambda_{n_{0}}=\frac{B(0)}{2 A(0)} .
$$

Remark 3.1. This situation of having one special eigenvalue might (only) happen for a special geometry of the underlying domain, therefore the situation in the first case is called generic.

Computing

$$
\begin{gathered}
4 A(0) C(0)-B^{2}(0)=0, \\
\left(l_{1} l_{2}-l_{3}\right)\left(n_{0}, 0\right)=0
\end{gathered}
$$

implies the existence of a value $\omega$ with real part equal to zero, by a generalization of the Routh-Hurwitz criterion given in [24]. Hence, since $\omega=0$ is not admissible, there exists an oscillating solution, so we do not have exponential stability in this special case.

Theorem 3.2. In the limit case of (1.5) there exist cases such that the asymptotic stability is not given.

Remark 3.3. For the generic case we expect exponential stability. 


\section{Spectral analysis for the limit case of (1.6)}

In this section we analyse the point spectrum of the equation (1.6) in the case that $\tau_{\nu}^{*}=k^{*} \tau_{q}$. We would like to prove that we can find a positive $\epsilon$ such that the point spectrum is on the left of the line $\operatorname{Re}\{\omega\}=-\epsilon$. This suggests the exponential decay of the solutions.

We first note that our equation becomes

$$
\ddot{T}+\tau_{1} \dddot{T}=k^{*} \triangle T+k^{*} \tau_{1} \triangle \dot{T}+k \tau_{2} \triangle \ddot{T}
$$

where

$$
\tau_{1}=\tau_{q} \quad \text { and } \quad \tau_{2}=\tau_{T} .
$$

If we look for solutions of the form (2.2), the parameter $\omega$ must satisfy the equation

$$
\tau_{1} \omega^{3}+\left(1+k \tau_{2} \lambda_{n}\right) \omega^{2}+\tau_{1} k^{*} \lambda_{n} \omega+k^{*} \lambda_{n}=0 .
$$

Exchanging again $\omega$ by $z-\epsilon$ we obtain the equation (2.4) with

$$
\begin{gathered}
l_{1}=\tau_{1}^{-1}\left(1+k \tau_{2} \lambda_{n}\right)-3 \epsilon, \quad l_{2}=k^{*} \lambda_{n}-\frac{2\left(1+k \tau_{2} \lambda_{n}\right)}{\tau_{1}} \epsilon+3 \epsilon^{2}, \\
l_{3}=\frac{k^{*} \lambda_{n}}{\tau_{1}}-\epsilon^{3}+\frac{\epsilon^{2}\left(1+k \tau_{2} \lambda_{n}\right)}{\tau_{1}}-k^{*} \lambda_{n} \epsilon .
\end{gathered}
$$

Again, we can always take $\epsilon$ small enough to guarantee that $l_{i}>0$ for every $i=1,2,3$. We also have

$$
l_{1} l_{2}-l_{3}=\lambda_{n}^{2}\left(\frac{k k^{*} \tau_{2}}{\tau_{1}}+c_{1} \epsilon\right)+\lambda_{n}\left(c_{2} \epsilon+c_{3} \epsilon^{2}\right)+\left(c_{4} \epsilon+c_{5} \epsilon^{2}+c_{6} \epsilon^{3}\right),
$$

with constants $c_{j}, j=1, \ldots, 6$, that are independent of $\epsilon$ and of $n$.

Since $0<\lambda_{n} \rightarrow \infty$ (as $\left.n \rightarrow \infty\right)$, we conclude that

$$
\exists c_{0}>0 \exists \epsilon_{0}>0 \forall 0<\epsilon \leq \epsilon_{0} \forall n: l_{1} l_{2}-l_{3} \geq c_{0} .
$$

Thus, the point spectrum is strictly to the left of the imaginary axis. This suggests the exponential stability of the system, which will be proved rigorously in Section 7 .

Theorem 4.1. In the limit case of (1.6) the spectrum is located strictly left to the imaginary axis.

\section{Spectral analysis for the limit cases of (1.7)}

The exponential stability of solutions of equation (1.7) is known when $k^{*} \tau_{q}<\tau_{\nu}^{*}<\frac{2 k \tau_{T}}{\tau_{q}}$. Several possibilities should be considered as limit cases. We will study three cases. The first one corresponds to $k^{*} \tau_{q}=\tau_{\nu}^{*}<\frac{2 k \tau_{T}}{\tau_{q}}$, the second one will be when $k^{*} \tau_{q}<\tau_{\nu}^{*}=\frac{2 k \tau_{T}}{\tau_{q}}$, and the last one is $k^{*} \tau_{q}=\tau_{\nu}^{*}=\frac{2 k \tau_{T}}{\tau_{q}}$. 


\subsection{Case $k^{*} \tau_{q}=\tau_{\nu}^{*}<\frac{2 k \tau_{T}}{\tau_{q}}$}

We here will see that the point spectrum is strictly away from the imaginary axis when $k^{*} \tau_{q}=\tau_{\nu}^{*}<\frac{2 k \tau_{T}}{\tau_{q}}$, which suggests the exponential decay of solutions.

If $k^{*} \tau_{q}=\tau_{\nu}^{*}<\frac{2 k \tau_{T}}{\tau_{q}}$ equation (1.7) can be written as

$$
\ddot{T}+\tau_{1} \dddot{T}+\frac{\tau_{1}^{2}}{2} \dddot{T}=k^{*} \triangle T+k^{*} \tau_{1} \triangle \dot{T}+k \tau_{2} \triangle \ddot{T},
$$

where $\tau_{1}=\tau_{q}$ and $\tau_{2}=\tau_{T}$. If we look for solutions of the form (2.2), the parameter $\omega$ must satisfy the equation

$$
\frac{\tau_{1}^{2}}{2} \omega^{4}+\tau_{1} \omega^{3}+\left(1+k \tau_{2} \lambda_{n}\right) \omega^{2}+\tau_{1} k^{*} \lambda_{n} \omega+k^{*} \lambda_{n}=0 .
$$

We will see that in this case we can find $\epsilon>0$ such that the point spectrum is on the left of the line $\operatorname{Re}\{\omega\}=-\epsilon$. The change of $\omega$ to $z-\epsilon$ leads to the equation

$$
z^{4}+a_{1} z^{3}+a_{2} z^{2}+a_{3} z+a_{4}=0
$$

where

$$
\begin{gathered}
a_{1}=\frac{2}{\tau_{1}}-4 \epsilon, a_{2}=\frac{2\left(1+\tau_{2} k \lambda_{n}\right)}{\tau_{1}^{2}}+6 \epsilon^{2}-\frac{6 \epsilon}{\tau_{1}}, \\
a_{3}=\frac{2 k^{*} \lambda_{n}}{\tau_{1}}-4 \epsilon^{3}+\frac{6 \epsilon^{2}}{\tau_{1}}-\frac{4 \epsilon\left(1+k \tau_{2} \lambda_{n}\right)}{\tau_{1}^{2}} \\
a_{4}=\frac{2 k^{*} \lambda_{n}}{\tau_{1}^{2}}+\epsilon^{4}-\frac{2 \epsilon^{3}}{\tau_{1}}+\frac{2 \epsilon^{2}\left(1+k \tau_{2} \lambda_{n}\right)}{\tau_{1}^{2}}-\frac{2 k^{*} \lambda_{n} \epsilon}{\tau_{1}} .
\end{gathered}
$$

Considering again the Hurwitz criterium, we know that the solutions have negative real parts whenever

$$
a_{i}>0, \quad i=1,2,3,4, \quad a_{1} a_{2}-a_{3}>0, \quad \text { and } a_{1} a_{2} a_{3}-a_{1}^{2} a_{4}-a_{3}^{2}>0 .
$$

In our case, we immediately see that all the coefficients $a_{j}, j=1,2,3,4$, are positive whenever $\epsilon$ is small enough. Let us consider the other two conditions in (5.4). First, it is

$$
a_{1} a_{2}-a_{3}=\frac{4}{\tau_{1}^{3}}+\underbrace{\left(\frac{4 k \tau_{2}-2 \tau_{1}^{2} k^{*}-4 \tau_{1} \tau_{2} k \epsilon}{\tau_{1}^{3}}\right)}_{=: D} \lambda_{n}+c_{1} \epsilon+c_{2} \epsilon^{2}+c_{3} \epsilon^{3},
$$

where $c_{j}, j=1,2,3$, and also $c_{4}, \ldots, c_{7}$ in the sequel, denote constants not depending on $\epsilon$ nor on $n$.

Since

$$
\tau_{1}^{3} D=2\left(2 \tau_{2} k-\tau_{1}^{2} k^{*}\right)+c_{4} \epsilon
$$


and $2 \tau_{2} k-\tau_{1}^{2} k^{*}>0$ by our assumption, we conclude that $D>0$ for sufficiently small $\epsilon$, hence also

$$
a_{1} a_{2}-a_{3}>0
$$

for sufficiently small $\epsilon$. Finally, we have

$$
F:=a_{1} a_{2} a_{3}-a_{1}^{2} a_{4}-a_{3}^{2}=\lambda_{n}^{2}\left(\frac{8 \tau_{2} k k^{*}}{\tau_{1}^{4}}-\frac{4\left(k^{*}\right)^{2}}{\tau_{1}^{2}}+c_{5} \epsilon \Psi_{1}(\epsilon)\right)+\lambda_{n} c_{6} \epsilon \Psi_{2}(\epsilon)+c_{7} \epsilon \Psi_{3}(\epsilon)
$$

with smooth functions $\Psi_{j}, j=1,2,3$, satisfying

$$
\sup _{0 \leq \epsilon \leq 1} \Psi_{j}(\epsilon)<\infty
$$

Since

$$
\frac{8 \tau_{2} k k^{*}}{\tau_{1}^{4}}-\frac{4\left(k^{*}\right)^{2}}{\tau_{1}^{2}}=\frac{4 k^{*}}{\tau_{1}^{2}}\left(\frac{2 \tau_{2} k}{\tau_{1}^{2}}-k^{*}\right)
$$

and $\frac{2 \tau_{2} k}{\tau_{1}^{2}}-k^{*}>0$ holds by our assumption in this limit case, we conclude that $F>0$ for sufficiently small $\epsilon$. Hence, the real parts of the zeros are strictly away from the imaginary axis. This suggests the exponential stability of the system, which will be proved rigorously in Section 8.

Theorem 5.1. In the first limit case of (1.7) the spectrum is located strictly left to the imaginary axis.

\subsection{Case $k^{*} \tau_{q}<\tau_{\nu}^{*}=\frac{2 k \tau_{T}}{\tau_{q}}$}

Now, we would like to prove that when $k^{*} \tau_{q}<\tau_{\nu}^{*}=\frac{2 k \tau_{T}}{\tau_{q}}$, there are always elements in the point spectrum as near as we want to the imaginary axis. If we substitute $\tau_{\nu}^{*}$ by $\frac{2 k \tau_{T}}{\tau_{q}}$ in equation (1.7) and consider solutions of the form (2.2), we see that $\omega$ must satisfy the equation

$$
\frac{\tau_{1}^{3}}{2} \omega^{4}+\tau_{1}^{2} \omega^{3}+\tau_{1}\left(1+k \tau_{2} \lambda_{n}\right) \omega^{2}+2 k \tau_{2} \lambda_{n} \omega+k^{*} \tau_{1} \lambda_{n}=0
$$

Here we use the same notation as in the previous sub-section. The change of $\omega$ to $z-\epsilon$ leads to the equation (5.3) with

$$
\begin{gathered}
a_{1}=\frac{2}{\tau_{1}}-4 \epsilon, a_{2}=\frac{2\left(1+k \tau_{2} \lambda_{n}\right)}{\tau_{1}^{2}}+6 \epsilon^{2}-\frac{6 \epsilon}{\tau_{1}}, \\
a_{3}=\frac{4 k \tau_{2} \lambda_{n}}{\tau_{1}^{3}}-4 \epsilon^{3}+\frac{6 \epsilon^{2}}{\tau_{1}}-\frac{4 \epsilon\left(1+k \tau_{2} \lambda_{n}\right)}{\tau_{1}^{2}}, \\
a_{4}=\frac{2 k^{*} \lambda_{n}}{\tau_{1}^{2}}+\epsilon^{4}-\frac{2 \epsilon^{3}}{\tau_{1}}+\frac{2\left(1+k \tau_{2} \lambda_{n}\right) \epsilon^{2}}{\tau_{1}^{2}}-\frac{4 k \tau_{2} \lambda_{n} \epsilon}{\tau_{1}^{3}} .
\end{gathered}
$$


It is obvious that $a_{j}>0, j=1,2,3,4$ for sufficiently small $\epsilon$.

On the other hand, we have

$$
H:=a_{1} a_{2}-a_{3}=\frac{4}{\tau_{1}^{3}}-\frac{4 \tau_{2} k}{\tau_{1}^{2}} \epsilon \lambda_{n}+c_{1} \epsilon+c_{2} \epsilon^{2}+c_{3} \epsilon^{3},
$$

with constants $c_{j}, j=1,2,3$, being independent of $\epsilon$ and $n$. The term $H$ is not uniformly (in $n$ ) positive, since for any however small positive $\epsilon$, the term which is linear in $\lambda_{n}$ tends to $-\infty$ as $n \rightarrow \infty$. This implies the non-exponential stability, i.e. a slow decay.

Theorem 5.2. In the second limit case of (1.7) we have slow decay in general.

\subsection{Case $k^{*} \tau_{q}=\tau_{\nu}^{*}=\frac{2 k \tau_{T}}{\tau_{q}}$}

We can regard this case as a limit case of the second case when $k^{*} \tau_{q}=\frac{2 k \tau_{T}}{\tau_{q}}$. Thus, we also see in this case that $a_{1} a_{2}-a_{3}$ will be negative for any fixed positive $\epsilon$ and for sufficiently large $n$ (similarly for $a_{1} a_{2} a_{3}-a_{1}^{2}-a_{3}^{2}$ ). Hence we get

Theorem 5.3. In the third limit case of (1.7) we have slow decay in general.

\section{Well-posedness for (three-)phase-lag heat conduc- tion}

We consider the phase-lag flux-temperature relation (1.2), i.e.

$$
q\left(x, t+\tau_{q}\right)=-\left(k \nabla T\left(x, t+\tau_{T}\right)+k^{*} \nabla \nu\left(x, t+\tau_{\nu}\right)\right)
$$

with $k \geq 0$ and $k^{*}>0$. Well-posedness results in the case of dual-phase-lag with $k>0$ and $k^{*}=0$ have been given in $[15,17,23]$. Now let $k^{*}>0$, but possibly $k=0$ (i.e. real three-phase-lag, or dual-phase-lag different from the case $\left.k^{*}=0\right)$.

Formal Taylor approximations in $\tau_{q}, \tau_{T}$ and $\tau_{\nu}$, respectively, in (6.1) yield the general form

$$
\sum_{j=0}^{m_{q}} \frac{\tau_{q}^{j}}{j !} \frac{\partial^{j}}{\partial t^{j}} q(x, t)=-k \sum_{j=0}^{m_{T}} \frac{\tau_{T}^{j}}{j !} \frac{\partial^{j}}{\partial t^{j}} \nabla T(x, t)-k^{*} \sum_{j=0}^{m_{\nu}} \frac{\tau_{\nu}^{j}}{j !} \frac{\partial^{j}}{\partial t^{j}} \nabla \nu(x, t)
$$

with $m_{q}, m_{T}, m_{\nu} \in \mathbb{N}$. When combined with the energy equation (1.3) it leads to

$$
\sum_{j=0}^{m_{q}} \frac{\tau_{q}^{j}}{j !} \frac{\partial^{j+2}}{\partial t^{j+2}} T(x, t)=\sum_{j=0}^{m} a_{j} \frac{\partial^{j}}{\partial t^{j}} \Delta T(x, t)
$$


where $m:=m_{\nu}$ if $k=0$, and $m:=\max \left\{m_{T}+1, m_{\nu}\right\}$ if $k>0$. The coefficients $a_{j}>0$ are given by $a_{j}:=\frac{\tau_{\nu}^{j}}{j !} k^{*}$ for $j=0, \ldots, m$ if $k=0$ and otherwise $a_{0}:=k^{*}$ and

$$
\begin{aligned}
\text { if } \quad m_{\nu} \leq m_{T}: & a_{j}:=\frac{\tau_{T}^{j-1}}{(j-1) !} k+\frac{\tau_{\nu}^{j}}{j !} k^{*} & \text { for } \quad j=1, \ldots, m_{\nu}, \\
& a_{j}:=\frac{\tau_{T}^{j-1}}{(j-1) !} k & \text { for } \quad j=m_{\nu}+1, \ldots, m, \\
\text { if } \quad m_{\nu}=m_{T}+1: & a_{j}:=\frac{\tau_{T}^{j-1}}{(j-1) !} k+\frac{\tau_{\nu}^{j}}{j !} k^{*} & \text { for } \quad j=1, \ldots, m, \\
\text { if } \quad m_{\nu}>m_{T}+1: & a_{j}:=\frac{\tau_{T}^{j-1}}{(j-1) !} k+\frac{\tau_{\nu}^{j}}{j !} k^{*} & \text { for } \quad j=1, \ldots, m_{T}+1, \\
& a_{j}:=\frac{\tau_{\nu}^{j}}{j !} k^{*} & \text { for } \quad j=m_{T}+2, \ldots, m .
\end{aligned}
$$

In this notation the equations (1.6) and (1.7) correspond to the cases (a) $m_{q}=1, m_{T}=$ $1, m_{\nu}=1$ and (b) $m_{q}=2, m_{T}=1, m_{\nu}=1$, respectively.

If $m_{q} \geq m+1$ the problem (6.3) is ill-posed (see [3]), which, e.g., applies to the case (b) with $k=0$ (special dual-phase-lag). We will now show well-posedness results for (6.3) for the following two cases: (i) $m_{q}=1, m=2$ and (ii) $m_{q}=m$, whereby the three-phase-lag problems (1.6) and (1.7) with $k>0$, as well as the special dual-phase-lag problem (1.6) with $k=0$, are included.

\subsection{Well-posedness in case of $m_{q}=1, m=2$}

Let $\Omega \subset \mathbb{R}^{n}$ be bounded. We are looking for a solution $T: \Omega \times[0, \infty) \rightarrow \mathbb{R}$ to the initial boundary value problem,

$$
\begin{gathered}
\ddot{T}+\tau_{q} \dddot{T}=k^{*} \Delta T+a_{1} \Delta \dot{T}+a_{2} \Delta \ddot{T} \quad \text { in } \Omega \times(0, \infty), \\
T(\cdot, 0)=\theta_{0}, \quad \dot{T}(\cdot, 0)=\theta_{1}, \quad \ddot{T}(\cdot, 0)=\theta_{2} \quad \text { in } \Omega, \\
\left.T(\cdot, t)\right|_{\partial \Omega}=0 \quad \text { for } t \in[0, \infty),
\end{gathered}
$$

where the initial data $\theta_{0}: \Omega \rightarrow \mathbb{R}, \theta_{1}: \Omega \rightarrow \mathbb{R}$ and $\theta_{2}: \Omega \rightarrow \mathbb{R}$ are given.

We choose

$$
\mathcal{H}:=H_{0}^{1}(\Omega) \times H_{0}^{1}(\Omega) \times L^{2}(\Omega)
$$

and for $V, W \in \mathcal{H}$ we define

$$
\langle V, W\rangle_{\mathcal{H}}:=\left\langle\nabla V_{1}, \nabla W_{1}\right\rangle+a_{1}\left\langle\nabla V_{2}, \nabla W_{2}\right\rangle+\tau_{q}\left\langle V_{3}, W_{3}\right\rangle
$$

Here, $\langle\cdot, \cdot\rangle$ denotes the $L^{2}(\Omega)$-inner product with associated norm $\|\cdot\| \cdot\left(\mathcal{H},\langle\cdot, \cdot\rangle_{\mathcal{H}}\right)$ can be shown to be a Hilbert space, and the norm $\|\cdot\|_{\mathcal{H}}$ is equivalent to the norm $\|\cdot\|_{H_{0}^{1} \times H_{0}^{1} \times L^{2}}$. 
Transforming (6.4)-(6.6) to a first order system via $\mathcal{V}:=(T, \dot{T}, \ddot{T})$, we obtain

$$
\frac{d}{d t} \mathcal{V}(t)=\mathcal{A} \mathcal{V}(t)+\mathcal{F}(t), \quad \mathcal{V}(0)=\mathcal{V}_{0}
$$

where the operator $\mathcal{A}: D(\mathcal{A}) \subseteq \mathcal{H} \rightarrow \mathcal{H}$ is defined by

$$
D(\mathcal{A}):=\left\{V \in\left(H_{0}^{1}(\Omega)\right)^{3} \mid k^{*} \Delta V_{1}+a_{1} \Delta V_{2}+a_{2} \Delta V_{3} \in L^{2}(\Omega)\right\},
$$

and

$$
\mathcal{A} V:=\left(\begin{array}{ccc}
0 & 1 & 0 \\
0 & 0 & 1 \\
\frac{k^{*}}{\tau_{q}} \Delta & \frac{a_{1}}{\tau_{q}} \Delta & \frac{a_{2}}{\tau_{q}} \Delta-\frac{1}{\tau_{q}}
\end{array}\right) V \quad \text { for } V \in D(\mathcal{A}) .
$$

The corresponding initial condition is given by $\mathcal{V}_{0}:=\left(\theta_{0}, \theta_{1}, \theta_{2}\right)^{\prime}$. Besides, we have added a possible inhomogeneity $\mathcal{F}:[0, \infty) \rightarrow \mathcal{H}$, which does not affect the essential considerations concerning the existence of solutions.

Lemma 6.1. $\mathcal{A}$ is a closed linear operator with $\overline{D(\mathcal{A})}=\mathcal{H}$.

Proof. $\overline{D(\mathcal{A})}=\mathcal{H}$ is obvious by a density argument. Let $\left(V^{n}\right)_{n \in \mathbb{N}} \subseteq D(\mathcal{A})$ and $V, W \in \mathcal{H}$ with $V^{n} \stackrel{\mathcal{H}}{\rightarrow} V$ and $\mathcal{A} V^{n} \stackrel{\mathcal{H}}{\rightarrow} W$ as $n \rightarrow \infty$. Particularly, for the first component we have $V_{1}^{n} \stackrel{H_{0}^{1}}{\longrightarrow} V_{1} \in H_{0}^{1}(\Omega)$, remembering the equivalence of $\|\cdot\|_{\mathcal{H}}$ and $\|\cdot\|_{H_{0}^{1} \times H_{0}^{1} \times L^{2}} . V_{2}=W_{1}$ in $H_{0}^{1}(\Omega)$ results from $V_{2}^{n} \stackrel{H_{0}^{1}}{\longrightarrow} V_{2} \in H_{0}^{1}(\Omega)$ and $V_{2}^{n}=\left(\mathcal{A} V^{n}\right)_{1} \stackrel{H_{0}^{1}}{\longrightarrow} W_{1} \in H_{0}^{1}(\Omega)$. Since $V_{3}^{n} \stackrel{L^{2}}{\rightarrow} V_{3} \in L^{2}(\Omega)$ and $V_{3}^{n}=\left(\mathcal{A} V^{n}\right)_{2} \stackrel{H_{0}^{1}}{\longrightarrow} W_{2} \in L^{2}(\Omega)$ we conclude $V_{3}=W_{2}$ in $L^{2}(\Omega)$, and, by testing with $\nabla \varphi$ for $\varphi \in C_{0}^{\infty}(\Omega)$, one sees that the equality also holds in $H_{0}^{1}(\Omega)$. If we choose $\Phi=\left(0,0, \Phi_{3}\right) \in \mathcal{H}$ with $\Phi_{3} \in C_{0}^{\infty}(\Omega)$ being arbitrary, then, by definition of $\mathcal{A}$ and $\langle\cdot, \cdot\rangle_{\mathcal{H}}$,

$$
\left\langle\mathcal{A} V^{n}, \Phi\right\rangle_{\mathcal{H}} \longrightarrow\langle W, \Phi\rangle_{\mathcal{H}}=\tau_{q}\left\langle W_{3}, \Phi_{3}\right\rangle
$$

and

$$
\begin{aligned}
\left\langle\mathcal{A} V^{n}, \Phi\right\rangle_{\mathcal{H}} & =\left\langle k^{*} \Delta V_{1}^{n}+a_{1} \Delta V_{2}^{n}+a_{2} \Delta V_{3}^{n}-V_{3}^{n}, \Phi_{3}\right\rangle \\
& \longrightarrow-\left\langle k^{*} \nabla V_{1}+a_{1} \nabla V_{2}+a_{2} \nabla V_{3}, \nabla \Phi_{3}\right\rangle-\left\langle V_{3}, \Phi_{3}\right\rangle
\end{aligned}
$$

as $n \rightarrow \infty$. This implies $k^{*} \Delta V_{1}+a_{1} \Delta V_{2}+a_{2} \Delta V_{3} \in L^{2}(\Omega)$ with $k^{*} \Delta V_{1}+a_{1} \Delta V_{2}+a_{2} \Delta V_{3}=$ $\tau_{q} W_{3}+V_{3}$. Altogether we have shown $V \in D(\mathcal{A})$ and $\mathcal{A} V=W$, so $\mathcal{A}$ is closed.

Lemma 6.2. There exists $d>0$ such that $\mathcal{A}-\lambda$ is dissipative for all $\lambda>d$. In this case $\lambda-\mathcal{A}$ is injective, therefore invertible, with $(\lambda-\mathcal{A})^{-1}$ being continuous and closed.

Proof. For $V \in D(\mathcal{A})$ the appropriate choice of the inner product guarantees

$$
\begin{aligned}
\operatorname{Re}\langle\mathcal{A} V, V\rangle_{\mathcal{H}} & =\operatorname{Re}\left\langle\nabla V_{2}, \nabla V_{1}\right\rangle-k^{*} \operatorname{Re}\left\langle\nabla V_{1}, \nabla V_{3}\right\rangle-a_{2}\left\|\nabla V_{3}\right\|^{2}-\left\|V_{3}\right\|^{2} \\
& \leq\left(\frac{1}{2}\left\|\nabla V_{2}\right\|^{2}+\frac{1}{2}\left\|\nabla V_{1}\right\|^{2}\right)+k^{*}\left(\frac{1}{2 \epsilon}\left\|\nabla V_{1}\right\|^{2}+\frac{\epsilon}{2}\left\|\nabla V_{3}\right\|^{2}\right)-a_{2}\left\|\nabla V_{3}\right\|^{2}
\end{aligned}
$$


where $\epsilon>0$ is arbitrary. If we choose $\epsilon:=\frac{2 a_{2}}{k^{*}}$ and define $d:=\frac{1}{2} \max \left\{1+\frac{\left(k^{*}\right)^{2}}{2 a_{2}}, \frac{1}{a_{1}}\right\}$ we have

$$
\operatorname{Re}\langle\mathcal{A} V, V\rangle_{\mathcal{H}} \leq d\left(\left\|\nabla V_{1}\right\|^{2}+a_{1}\left\|\nabla V_{2}\right\|^{2}\right) \leq d\|V\|_{\mathcal{H}}^{2}
$$

which provides the dissipativity of $\mathcal{A}-\lambda$ for $\lambda>d$. This means that $\|(\lambda-\mathcal{A}) V\|_{\mathcal{H}} \geq$ $\lambda\|V\|_{\mathcal{H}}$ holds for all $V \in \mathcal{H}$ and $\lambda>d$, from which follows that $\lambda-\mathcal{A}$ is injective and $(\lambda-\mathcal{A})^{-1}$ is continuous. The latter operator is closed because of the closedness of $\mathcal{A}$.

Lemma 6.3. For $\lambda>d$ the operator $\lambda-\mathcal{A}$ is surjective, that is, its range is $R(\lambda-\mathcal{A})=\mathcal{H}$.

Proof. First let $F \in \mathcal{H}$ with the additional condition $F_{1}, F_{2} \in H^{2}(\Omega) \cap H_{0}^{1}(\Omega)$. By definition of $\mathcal{A}$ the problem $(\lambda-\mathcal{A}) V=F$ is equivalent to

$$
\left\{\begin{aligned}
\lambda V_{1}-V_{2} & =F_{1}, \\
\lambda V_{2}-V_{3} & =F_{2}, \\
\lambda V_{3}-\frac{k^{*}}{\tau_{q}} \Delta V_{1}-\frac{a_{1}}{\tau_{q}} \Delta V_{2}-\frac{a_{2}}{\tau_{q}} \Delta V_{3}+\frac{1}{\tau_{q}} V_{3} & =F_{3} .
\end{aligned}\right.
$$

Eliminating $V_{2}$ and $V_{3}$ leads to

$$
-\underbrace{\left(\frac{k^{*}}{\tau_{q}}+\frac{a_{1}}{\tau_{q}} \lambda+\frac{a_{2}}{\tau_{q}} \lambda^{2}\right)}_{=: \alpha} \Delta V_{1}+\underbrace{\left(\lambda^{3}+\frac{\lambda^{2}}{\tau_{q}}\right)}_{=: \beta} V_{1}=f
$$

with $f:=\left(\lambda^{2}+\frac{\lambda}{\tau_{q}}\right) F_{1}-\left(\frac{a_{1}}{\tau_{q}}+\frac{a_{2}}{\tau_{q}} \lambda\right) \Delta F_{1}+\left(\lambda+\frac{1}{\tau_{q}}\right) F_{2}-\frac{a_{2}}{\tau_{q}} \Delta F_{2}+F_{3}$, which is in $L^{2}(\Omega)$ because of the choice of $F$. The coefficients fulfill $\alpha, \beta>0$. By the Lax-Milgram theorem there uniquely exists $V_{1} \in H_{0}^{1}(\Omega)$ with $\Delta V_{1} \in L^{2}(\Omega)$ which solves $-\alpha \Delta V_{1}+\beta V_{1}=f$. Then

$$
V:=\left(V_{1}, \lambda V_{1}-F_{1}, \lambda^{2} V_{1}-\lambda F_{1}-F_{2}\right)^{\prime}
$$

is in $D(\mathcal{A})$ and solves $(\lambda-\mathcal{A}) V=F$.

Omitting further requirements on its components any $F \in \mathcal{H}$ can still be approximated by a sequence $\left(F^{n}\right)_{n \in \mathbb{N}} \subseteq\left(H^{2}(\Omega) \cap H_{0}^{1}(\Omega)\right)^{2} \times L^{2}(\Omega)$. Using the continuity and closedness of $(\lambda-\mathcal{A})^{-1}$ we also obtain $F \in R(\lambda-\mathcal{A})$ for any $F \in \mathcal{H}$.

Because of the last two lemmata we can apply the Lumer-Phillips theorem concluding that $\mathcal{A}$ generates a $C_{0}$-semigroup on $\mathcal{H}$. Now, the well-posedness result for (6.7) reads as follows:

Theorem 6.4. If $\mathcal{V}_{0} \in D(\mathcal{A})$ and either $\mathcal{F} \in C^{0}([0, \infty), D(\mathcal{A}))$ or $\mathcal{F} \in C^{1}([0, \infty), \mathcal{H})$ then there is a unique solution $\mathcal{V} \in C^{0}([0, \infty), \mathcal{H}) \cap C^{0}((0, \infty), D(\mathcal{A})) \cap C^{1}((0, \infty), \mathcal{H})$ to (6.7). 


\subsection{Well-posedness in case of $m_{q}=m$}

Using the same approach as before, we can look at

$$
\begin{gathered}
\sum_{j=0}^{m} \frac{\tau_{q}^{j}}{j !} \frac{\partial^{j+2}}{\partial t^{j+2}} T=\sum_{j=0}^{m} a_{j} \frac{\partial^{j}}{\partial t^{j}} \Delta T \quad \text { in } \Omega \times(0, \infty), \\
\frac{\partial^{j}}{\partial t^{j}} T(\cdot, 0)=\theta_{j} \quad \text { in } \Omega \quad \text { for } j=0, \ldots, m+1, \\
\left.T(\cdot, t)\right|_{\partial \Omega}=0 \quad \text { for } t \in[0, \infty),
\end{gathered}
$$

where the initial data $\theta_{j}: \Omega \rightarrow \mathbb{R}$ are given.

$$
\mathcal{H}:=\underbrace{H_{0}^{1}(\Omega) \times \ldots \times H_{0}^{1}(\Omega)}_{(m+1) \text {-times }} \times L^{2}(\Omega)
$$

with

$$
\begin{aligned}
\langle V, W\rangle_{\mathcal{H}}:= & \frac{3 m}{a_{m}}\left(\sum_{j=1}^{m} a_{j-1}^{2}\left\langle\nabla V_{j}, \nabla W_{j}\right\rangle\right)+a_{m}\left\langle\nabla V_{m+1}, \nabla W_{m+1}\right\rangle+\frac{\tau_{q}^{m}}{m !}\left\langle V_{m+2}, W_{m+2}\right\rangle \\
& +\sum_{j=1}^{m} a_{j-1}\left(\left\langle\nabla V_{j}, \nabla W_{m+1}\right\rangle+\left\langle\nabla V_{m+1}, \nabla W_{j}\right\rangle\right)
\end{aligned}
$$

will turn out to be a suitable Hilbert space. Its norm $\|\cdot\|_{\mathcal{H}}$ is equivalent to the standard norm $\|\cdot\|_{H_{0}^{1} \times \ldots \times H_{0}^{1} \times L^{2}}$. The coefficients in front of the terms $\left\langle\nabla V_{j}, \nabla W_{j}\right\rangle$ are chosen large enough to guarantee the positive definiteness of the inner product. The underlying estimate is

$$
\begin{array}{r}
\sum_{j=1}^{m} a_{j-1}\left(\left\langle\nabla V_{j}, \nabla V_{m+1}\right\rangle+\left\langle\nabla V_{m+1}, \nabla V_{j}\right\rangle\right) \geq \\
-2 \sum_{j=1}^{m} a_{j-1}\left(\frac{1}{2 \epsilon_{j}}\left\|\nabla V_{j}\right\|^{2}+\frac{\epsilon_{j}}{2}\left\|\nabla V_{m+1}\right\|^{2}\right) \\
\text { with } \epsilon_{j}:=\frac{a_{m}}{2 m a_{j-1}} \quad \text { for } 1 \leq j \leq m \\
=-\frac{a_{m}}{2}\left\|\nabla V_{m+1}\right\|^{2}-\frac{2 m}{a_{m}} \sum_{j=1}^{m} a_{j-1}^{2}\left\|\nabla V_{j}\right\|^{2} .
\end{array}
$$

On $\mathcal{H}$ we now consider

$$
\frac{d}{d t} \mathcal{V}(t)=\mathcal{A} \mathcal{V}(t)+\mathcal{F}(t), \quad \mathcal{V}(0)=\mathcal{V}_{0}
$$

where again an inhomogeneity $\mathcal{F}$ is allowed. The operator $\mathcal{A}: D(\mathcal{A}) \subseteq \mathcal{H} \rightarrow \mathcal{H}$ is defined by

$$
D(\mathcal{A}):=\left\{V \in\left(H_{0}^{1}(\Omega)\right)^{m+2} \mid \sum_{j=1}^{m+1} a_{j-1} \Delta V_{j} \in L^{2}(\Omega)\right\}
$$


and

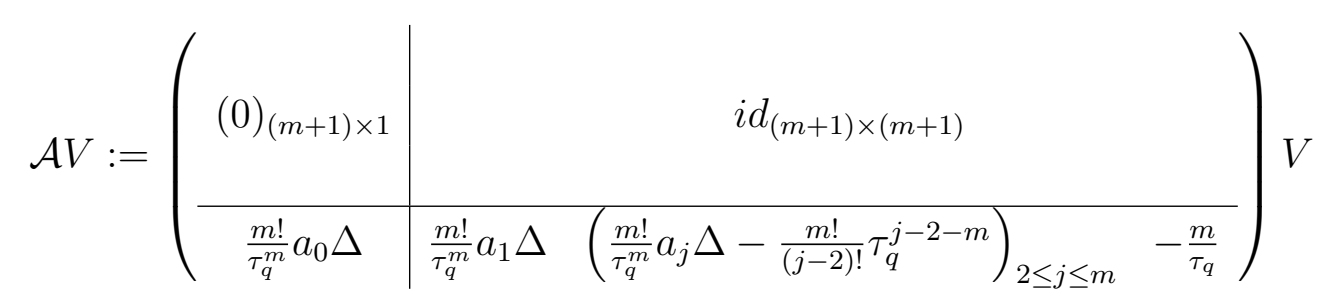

which is motivated by the transformation of (6.8)-(6.10) to a first order system via $\mathcal{V}:=$ $\left(T, \frac{\partial}{\partial t} T, \ldots, \frac{\partial^{m+1}}{\partial t^{m+1}} T\right)$. Accordingly the initial value is $\mathcal{V}_{0}:=\left(\theta_{0}, \theta_{1}, \ldots, \theta_{m+1}\right)^{\prime}$.

Lemma 6.5. $\mathcal{A}$ is a closed linear operator with $\overline{D(\mathcal{A})}=\mathcal{H}$.

Proof. $\overline{D(\mathcal{A})}=\mathcal{H}$ is true by a density argument. Let $\left(V^{n}\right)_{n \in \mathbb{N}} \subseteq D(\mathcal{A})$ and $V, W \in \mathcal{H}$ with $V^{n} \stackrel{\mathcal{H}}{\rightarrow} V$ and $\mathcal{A} V^{n} \stackrel{\mathcal{H}}{\rightarrow} W$ as $n \rightarrow \infty$. Because of the equivalence of $\|\cdot\|_{\mathcal{H}}$ and $\|\cdot\|_{H_{0}^{1} \times \ldots \times H_{0}^{1} \times L^{2}}$ the first component $V_{1}^{n}$ obviously tends to $V_{1} \in H_{0}^{1}(\Omega)$ as $n \rightarrow \infty$ and for $j=1, \ldots, m$ the identity $V_{j+1}=W_{j}$ in $H_{0}^{1}(\Omega)$ follows from

$$
V_{j+1}^{n} \stackrel{H_{0}^{1}}{\longrightarrow} V_{j+1} \in H_{0}^{1}(\Omega) \quad \text { and } \quad V_{j+1}^{n}=\left(\mathcal{A} V^{n}\right)_{j} \stackrel{H_{0}^{1}}{\longrightarrow} W_{j} \in H_{0}^{1}(\Omega) \quad \text { as } n \rightarrow \infty .
$$

The validity of $V_{m+2}=W_{m+1}$ in $H_{0}^{1}(\Omega)$ is ensured by

$$
V_{m+2}^{n} \stackrel{L^{2}}{\rightarrow} V_{m+2} \in L^{2}(\Omega) \quad \text { and } \quad V_{m+2}^{n}=\left(\mathcal{A} V^{n}\right)_{m+1} \stackrel{H_{0}^{1}}{\rightarrow} W_{m+1} \in H_{0}^{1}(\Omega) \quad \text { as } n \rightarrow \infty .
$$

For any $\Phi=\left(0, \ldots, 0, \Phi_{m+2}\right) \in \mathcal{H}$ with $\Phi_{m+2} \in C_{0}^{\infty}(\Omega)$ we obtain

$$
\left\langle\mathcal{A} V^{n}, \Phi\right\rangle_{\mathcal{H}} \longrightarrow\langle W, \Phi\rangle_{\mathcal{H}}=\frac{\tau_{q}^{m}}{m !}\left\langle W_{m+2}, \Phi_{m+2}\right\rangle
$$

and

$$
\begin{aligned}
\left\langle\mathcal{A} V^{n}, \Phi\right\rangle_{\mathcal{H}} & =\left\langle\sum_{j=0}^{m} a_{j} \Delta V_{j+1}^{n}-\sum_{j=2}^{m+1} \frac{\tau_{q}^{j-2}}{(j-2) !} V_{j+1}^{n}, \Phi_{m+2}\right\rangle \\
& \longrightarrow-\left\langle\sum_{j=0}^{m} a_{j} \nabla V_{j+1}, \nabla \Phi_{m+2}\right\rangle-\left\langle\sum_{j=2}^{m+1} \frac{\tau_{q}^{j-2}}{(j-2) !} V_{j+1}, \Phi_{m+2}\right\rangle
\end{aligned}
$$

by definition of $\mathcal{A}$ and $\langle\cdot, \cdot\rangle_{\mathcal{H}}$. This shows that $\sum_{j=0}^{m} a_{j} \Delta V_{j+1}$ is in $L^{2}(\Omega)$ fulfilling $\sum_{j=0}^{m} a_{j} \Delta V_{j+1}=\frac{\tau_{q}^{m}}{m !} W_{m+2}+\sum_{j=2}^{m+1} \frac{\tau_{q}^{j-2}}{(j-2) !} V_{j+1}$. So $\mathcal{A}$ is closed since $V \in D(\mathcal{A})$ and $\mathcal{A} V=W$.

Lemma 6.6. There exists $d>0$ such that $\mathcal{A}-\lambda$ is dissipative for all $\lambda>d$. In this case $\lambda-\mathcal{A}$ is injective with $(\lambda-\mathcal{A})^{-1}$ being continuous and closed. 
Proof. Let $V \in D(\mathcal{A})$. The form of the inner product enables

$$
\begin{aligned}
\operatorname{Re}\langle\mathcal{A} V, V\rangle_{\mathcal{H}}= & \frac{3 m}{a_{m}} \operatorname{Re} \sum_{j=1}^{m} a_{j-1}^{2}\left\langle\nabla V_{j+1}, \nabla V_{j}\right\rangle+\operatorname{Re} \sum_{j=1}^{m-1} a_{j-1}\left\langle\nabla V_{j+1}, \nabla V_{m+1}\right\rangle \\
& \quad-\operatorname{Re} \sum_{j=2}^{m} \frac{\tau_{q}^{j-2}}{(j-2) !}\left\langle V_{j+1}, V_{m+2}\right\rangle+a_{m-1}\left\|\nabla V_{m+1}\right\|^{2}-\frac{\tau_{q}^{m-1}}{(m-1) !}\left\|V_{m+2}\right\|^{2} \\
\leq & a_{m-1}\left\|\nabla V_{m+1}\right\|^{2}+\frac{3 m}{2 a_{m}} \sum_{j=1}^{m} a_{j-1}^{2}\left(\left\|\nabla V_{j+1}\right\|^{2}+\left\|\nabla V_{j}\right\|^{2}\right) \\
& +\sum_{j=1}^{m-1} \frac{a_{j-1}}{2}\left(\left\|\nabla V_{j+1}\right\|^{2}+\left\|\nabla V_{m+1}\right\|^{2}\right)+\frac{1}{2} \sum_{j=2}^{m} \frac{\tau_{q}^{j-2}}{(j-2) !}\left(\left\|V_{j+1}\right\|^{2}+\left\|V_{m+2}\right\|^{2}\right) \\
= & \frac{3 m}{2 a_{m}} a_{0}^{2}\left\|\nabla V_{1}\right\|^{2}+\sum_{j=2}^{m}\left(\frac{3 m}{2 a_{m}}\left(a_{j-2}^{2}+a_{j-1}^{2}\right)+\frac{a_{j-2}}{2}\right)\left\|\nabla V_{j}\right\|^{2}+\frac{1}{2} \sum_{j=2}^{m} \frac{\tau_{q}^{j-2}}{(j-2) !}\left\|V_{j+1}\right\|^{2} \\
& +\left(a_{m-1}+\frac{3 m}{2 a_{m}} a_{m-1}^{2}+\sum_{j=1}^{m-1} \frac{a_{j-1}}{2}\right)\left\|\nabla V_{m+1}\right\|^{2}+\left(\frac{1}{2} \sum_{j=2}^{m} \frac{\tau_{q}^{j-2}}{(j-2) !}\right)\left\|V_{m+2}\right\|^{2} \\
\leq & d_{1}\|V\|_{H_{0}^{1} \times \ldots \times H_{0}^{1} \times L^{2}} \\
\leq & d\|V\|_{\mathcal{H}}
\end{aligned}
$$

for some positive constants $d_{1}, d$. We conclude that $\mathcal{A}-\lambda$ is dissipative if $\lambda>d$. Then $(\lambda-\mathcal{A})^{-1}$ exists and it is continuous and closed.

Lemma 6.7. For $\lambda>d$ the operator $\lambda-\mathcal{A}$ is surjective, that is $R(\lambda-\mathcal{A})=\mathcal{H}$.

Proof. First let $F \in \mathcal{H}$ whose first $m$ components $F_{1}, \ldots, F_{m}$ are additionally assumed to be in $H^{2}(\Omega) \cap H_{0}^{1}(\Omega)$. The system $(\lambda-\mathcal{A}) V=F$, which is

$$
\left\{\begin{aligned}
\lambda V_{j}-V_{j+1} & =F_{j} \quad \text { for } j=1, \ldots, m+1, \\
\lambda V_{m+2}-\frac{m !}{\tau_{q}^{m}} \sum_{j=0}^{m} a_{j} \Delta V_{j+1}+\frac{m !}{\tau_{q}^{m}} \sum_{j=2}^{m+1} \frac{\tau_{q}^{j-2}}{(j-2) !} V_{j+1} & =F_{m+2}
\end{aligned}\right.
$$

can be transformed to a single equation by eliminating $V_{2}, \ldots, V_{m+2}$ via

$$
V_{j+1}=\lambda V_{j}-F_{j}=\lambda^{j} V_{1}-\sum_{k=1}^{j} \lambda^{j-k} F_{k} \quad \text { for } j=1, \ldots, m+1,
$$

according to the first $m+1$ equations. Thus we obtain

$$
-\underbrace{\left(\frac{m !}{\tau_{q}^{m}} \sum_{j=0}^{m} a_{j} \lambda^{j}\right)}_{=: \alpha} \Delta V_{1}+\underbrace{\left(\lambda^{m+2}+\frac{m !}{\tau_{q}^{m}} \sum_{j=2}^{m+1} \frac{\tau_{q}^{j-2}}{(j-2) !} \lambda^{j}\right)}_{=: \beta} V_{1}=f
$$


with $f:=F_{m+2}+\sum_{k=1}^{m+1} \lambda^{m+2-k} F_{k}-\frac{m !}{\tau_{q}^{m}} \sum_{j=1}^{m} a_{j} \sum_{k=1}^{j} \lambda^{j-k} \Delta F_{k}+\frac{m !}{\tau_{q}^{m}} \sum_{j=2}^{m+1} \frac{\tau_{q}^{j-2}}{(j-2) !} \sum_{k=1}^{j} \lambda^{j-k} F_{k}$.

We have $\alpha, \beta>0$, and the additional condition on $F$ ensures $f \in L^{2}(\Omega)$, so $-\alpha \Delta V_{1}+\beta V_{1}=$ $f$ has got a unique solution $V_{1} \in H_{0}^{1}(\Omega)$ with $\Delta V_{1} \in L^{2}(\Omega)$. We define

$$
V:=\left(V_{1}, \ldots, V_{m+2}\right)^{\prime} \quad \text { where } \quad V_{j+1}:=\lambda^{j} V_{1}-\sum_{k=1}^{j} \lambda^{j-k} F_{k} \quad \text { for } j \in\{1, \ldots, m+1\} .
$$

Then $V \in D(\mathcal{A})$, and $V$ solves $(\lambda-\mathcal{A}) V=F$.

As before we conclude $F \in R(\lambda-\mathcal{A})$ for any $F \in \mathcal{H}$ by a density argument using the continuity and closedness of $(\lambda-\mathcal{A})^{-1}$.

As a result of the last two lemmata (applying the Lumer-Phillips theorem) we obtain the well-posedness of (6.11):

Theorem 6.8. If $\mathcal{V}_{0} \in D(\mathcal{A})$ and either $\mathcal{F} \in C^{0}([0, \infty), D(\mathcal{A}))$ or $\mathcal{F} \in C^{1}([0, \infty), \mathcal{H})$, then there is a unique solution $\mathcal{V} \in C^{0}([0, \infty), \mathcal{H}) \cap C^{0}((0, \infty), D(\mathcal{A})) \cap C^{1}((0, \infty), \mathcal{H})$ to (6.11).

\section{Exponential stability for the limit case of (1.6)}

For the case that $\tau_{\nu}^{*}=k^{*} \tau_{q}$ in (1.6), we shall prove the exponential stability suggested by our spectral analysis, cf. Theorem 4.1. We recall the differential equation

$$
\ddot{T}+\tau_{1} \dddot{T}=k^{*} \triangle T+k^{*} \tau_{1} \triangle \dot{T}+k \tau_{2} \triangle \ddot{T}
$$

with $\tau_{1}=\tau_{q}$ and $\tau_{2}=\tau_{T}$, supplemented by initial and Dirichlet type boundary conditions. As in [18] we define the functions

$$
F(t):=\frac{1}{2} \int_{\Omega}\left(\left(\dot{T}+\tau_{1} \ddot{T}\right)^{2}+k^{*}\left|\nabla\left(T+\tau_{1} \dot{T}\right)\right|^{2}+k \tau_{2}|\nabla \dot{T}|^{2}\right)(x, t) d x,
$$

representing a non-negative energy functional, and

$$
G(t):=\int_{\Omega}\left(\frac{k^{*} \tau_{1}}{2}|\nabla T|^{2}+k \tau_{2} \nabla T \nabla \dot{T}+T \dot{T}+\tau_{1} T \ddot{T}\right)(x, t) d x .
$$

To treat the limit case, we need to introduce an additional function, in contrast to [18], namely,

$$
H(t):=-\int_{\Omega}(\nabla T \nabla \dot{T})(x, t) d x .
$$

As Lyapunov function we now consider $E$ defined by

$$
E(t):=F(t)+\epsilon^{3 / 4} H(t)+\epsilon G(t)
$$


where the positive $\epsilon$ will be chosen small enough in the sequel. For sufficiently small $\varepsilon$, we have that $E$ is equivalent to $F$, i.e.,

$$
\exists p_{1}, p_{2}>0 \forall t \geq 0: p_{1} F(t) \leq E(t) \leq p_{2} F(t) .
$$

Moreover, $F=F(t)$ is equivalent to $\int_{\Omega}\left(|\nabla T|^{2}+|\nabla \dot{T}|^{2}+|\ddot{T}|^{2}\right)(x, t) d x$.

We have, as in [18], that

$$
F^{\prime}(t)=-\int_{\Omega} k \tau_{1} \tau_{2}|\nabla \ddot{T}|^{2}(x, t) d x
$$

and

$$
G^{\prime}(t)=-k^{*} \int_{\Omega}|\nabla T|^{2}(x, t) d x+\int_{\Omega}\left(k \tau_{2}|\nabla \dot{T}|^{2}+|\dot{T}|^{2}+\tau_{1} \dot{T} \ddot{T}\right)(x, t) d x .
$$

Additionally, we now have

$$
H^{\prime}(t)=-\int_{\Omega} \nabla T \nabla \ddot{T}(x, t) d x-\int_{\Omega}|\nabla \dot{T}|^{2}(x, t) d x .
$$

Thus we get

$$
\begin{aligned}
E^{\prime}(t)= & -\int_{\Omega} k \tau_{1} \tau_{2}|\nabla \ddot{T}|^{2}(x, t) d x-\epsilon k^{*} \int_{\Omega}|\nabla T|^{2}(x, t) d x-\left(\epsilon^{3 / 4}-\epsilon k \tau_{2}\right) \int_{\Omega}|\nabla \dot{T}|^{2}(x, t) d x \\
& +\epsilon \int_{\Omega}\left(\dot{T}^{2}+\tau_{1} \dot{T} \ddot{T}\right)(x, t) d x-\epsilon^{3 / 4} \int_{\Omega}(\nabla T \nabla \ddot{T})(x, t) d x .
\end{aligned}
$$

This implies

$$
\begin{aligned}
E^{\prime}(t) \leq & -\int_{\Omega}\left(k \tau_{1} \tau_{2}-\frac{\epsilon \tau_{1}}{2 \lambda_{1}}\right)|\nabla \ddot{T}|^{2}(x, t) d x-\epsilon k^{*} \int_{\Omega}|\nabla T|^{2}(x, t) d x \\
& -\left(\epsilon^{3 / 4}-\epsilon k \tau_{2}-\frac{\epsilon}{\lambda_{1}}-\frac{\epsilon \tau_{1}}{2 \lambda_{1}}\right) \int_{\Omega}|\nabla \dot{T}|^{2}(x, t) d x \\
& -\epsilon^{3 / 4} \int_{\Omega} \nabla T \nabla \ddot{T}(x, t) d x .
\end{aligned}
$$

Noting that the matrix

$$
M:=\left(\begin{array}{ccc}
\left(k \tau_{1} \tau_{2}-\frac{\epsilon \tau_{1}}{2 \lambda_{1}}\right) & 0 & \frac{\epsilon^{3 / 4}}{2} \\
0 & \left(\epsilon^{3 / 4}-\epsilon k \tau_{2}-\frac{\epsilon}{\lambda_{1}}-\frac{\epsilon \tau_{1}}{2 \lambda_{1}}\right) & 0 \\
\frac{\epsilon^{3 / 4}}{2} & 0 & \epsilon k^{*}
\end{array}\right)
$$

is positive definite whenever $\epsilon$ is sufficiently small, we obtain, using (7.4), with positive constants $c, c_{1}$,

$$
E^{\prime}(t) \leq-c F(t) \leq-c_{1} E(t)
$$

implying the exponential decay of $E(t)$, and hence of $F(t)$. That is we have proved

Theorem 7.1. In the limit case of (1.6), we have exponential energy decay, i.e.,

$$
\exists c_{1}, c_{2}>0 \forall t \geq 0: \quad F(t) \leq c_{2} \exp \left(-c_{1} t\right) F(0) .
$$




\section{Exponential stability for a limit case of (1.7)}

For the case that $k^{*} \tau_{q}=\tau_{\nu}^{*}<\frac{2 k \tau_{T}}{\tau_{q}}$ in (1.7), we shall prove the exponential stability suggested by our spectral analysis, cf. Theorem 5.1. We recall the differential equation

$$
\ddot{T}+\tau_{1} \dddot{T}+\frac{\tau_{1}^{2}}{2} \dddot{T}=k^{*} \triangle T+k^{*} \tau_{1} \triangle \dot{T}+k \tau_{2} \triangle \ddot{T},
$$

with $\tau_{1}=\tau_{q}$ and $\tau_{2}=\tau_{T}$, supplemented by initial and Dirichlet type boundary conditions. As in [18] we define the function

$$
\begin{aligned}
& F(t):=\frac{1}{2} \int_{\Omega}\left(\left(\dot{T}+\tau_{1} \ddot{T}+\frac{\tau_{1}^{2}}{2} \dddot{T}\right)^{2}+k^{*}\left|\nabla\left(T+\tau_{1} \dot{T}+\frac{\tau_{1}^{2}}{2} \ddot{T}\right)\right|^{2}\right. \\
& \left.+\left(k \tau_{2}-\frac{k^{*} \tau_{1}^{2}}{2}\right)|\nabla \dot{T}|^{2}+\frac{\tau_{1}^{2}}{2}\left(k \tau_{2}-\frac{k^{*} \tau_{1}^{2}}{2}\right)|\nabla \ddot{T}|^{2}\right)(x, t) d x .
\end{aligned}
$$

It is easy to see that $F=F(t)$ is equivalent to

$$
N(t):=\int_{\Omega}\left(|\nabla T|^{2}+|\nabla \dot{T}|^{2}+|\nabla \ddot{T}|^{2}+|\dddot{T}|^{2}\right)(x, t) d x
$$

i.e.,

$$
\exists p_{1}, p_{2}>0 \forall t \geq 0: p_{1} F(t) \leq N(t) \leq p_{2} F(t) .
$$

We have

$$
F^{\prime}(t)=-\tau_{1}\left(k \tau_{2}-\frac{k^{*} \tau_{1}^{2}}{2}\right) \int_{\Omega}|\nabla \ddot{T}|^{2}(x, t) d x .
$$

We also define, as in [18],

$$
G(t):=\frac{1}{2} \int_{\Omega}\left(\frac{\tau_{1}^{2}}{2}|\dddot{T}|^{2}+|\ddot{T}|^{2}+k \tau_{2}|\nabla \ddot{T}|^{2}+2 k^{*} \tau_{1} \nabla \dot{T} \nabla \ddot{T}+2 k^{*} \nabla T \nabla \ddot{T}\right)(x, t) d x
$$

which satisfies

$$
G^{\prime}(t)=-\tau_{1} \int_{\Omega}|\dddot{T}(x, t)|^{2} d x+\int_{\Omega}\left(k^{*} \tau_{1}|\nabla \ddot{T}|^{2}+k^{*} \nabla \dot{T} \nabla \ddot{T}\right)(x, t) d x .
$$

As a new function, we define

$$
H_{\delta}(t):=\int_{\Omega}\left(\frac{k^{*} \tau_{1}}{2}|\nabla T|^{2}+\left(k \tau_{2}-\delta\right) \nabla T \nabla \dot{T}+T \dot{T}+\tau_{1} T \ddot{T}+\frac{\tau_{1}^{2}}{2} T \dddot{T}\right)(x, t) d x
$$

where $\delta$ is a positive constant to be chosen later (large enough). We have

$$
\begin{aligned}
H_{\delta}^{\prime}(t)= & -k^{*} \int_{\Omega}|\nabla T|^{2}(x, t) d x+\left(k \tau_{2}-\delta\right) \int_{\Omega}|\nabla \dot{T}|^{2}(x, t) d x+\int_{\Omega}|\dot{T}|^{2}(x, t) d x \\
& +\tau_{1} \int_{\Omega}\left(\dot{T} \ddot{T}+\frac{\tau_{1}}{2} \ddot{T} \dddot{T}\right)(x, t) d x-\delta \int_{\Omega} \nabla T \nabla \ddot{T}(x, t) d x .
\end{aligned}
$$


Let $\epsilon$ be a positive number to be chosen below (small enough). We have

$$
\begin{aligned}
G^{\prime}(t)+\epsilon H_{\delta}^{\prime}(t)= & -\tau_{1} \int_{\Omega}|\dddot{T}|^{2}(x, t) d x-\epsilon k^{*} \int_{\Omega}|\nabla T|^{2}(x, t) d x \\
& +\epsilon\left(k \tau_{2}-\delta\right) \int_{\Omega}|\nabla \dot{T}|^{2}(x, t) d x \\
& +\int_{\Omega}\left(k^{*} \tau_{1}|\nabla \ddot{T}|^{2}+k^{*} \nabla \dot{T} \nabla \ddot{T}\right)(x, t) d x \\
& +\epsilon \int_{\Omega}|\dot{T}|^{2}(x, t) d x+\epsilon \tau_{1} \int_{\Omega}\left(\dot{T} \ddot{T}+\frac{\tau_{1}}{2} \dot{T} \dddot{T}\right)(x, t) d x \\
& -\epsilon \delta \int_{\Omega} \nabla T \nabla \ddot{T}(x, t) d x .
\end{aligned}
$$

We note that

$$
\begin{gathered}
\left|\int_{\Omega} k^{*} \nabla \dot{T} \nabla \ddot{T}(x, t) d x\right| \leq \frac{k^{*} \epsilon}{2} \int_{\Omega}|\nabla \dot{T}|^{2}(x, t) d x+\frac{k^{*}}{2 \epsilon} \int_{\Omega}|\nabla \ddot{T}|^{2}(x, t) d x \\
\epsilon \int_{\Omega}|\dot{T}|^{2}(x, t) d x \leq \frac{\epsilon}{\lambda_{1}} \int_{\Omega}|\nabla \dot{T}|^{2}(x, t) d x \\
\left|\epsilon \tau_{1} \int_{\Omega}\left(\dot{T} \ddot{T}+\frac{\tau_{1}}{2} \ddot{T} \dddot{T}\right)(x, t) d x\right| \leq \frac{2 \tau_{1}+\tau_{1}^{2}}{4 \lambda_{1}} \int_{\Omega}|\nabla \dot{T}|^{2}(x, t) d x+\epsilon \frac{\tau_{1}}{2 \lambda_{1}} \int_{\Omega}|\nabla \ddot{T}|^{2}(x, t) d x \\
+\epsilon \frac{\tau_{1}^{2}}{4} \int_{\Omega}|\dddot{T}|^{2}(x, t) d x
\end{gathered}
$$

and

$$
\left|\epsilon \delta \int_{\Omega} \nabla T \nabla \ddot{T}(x, t) d x\right| \leq \epsilon^{2} \frac{\delta}{2} \int_{\Omega}|\nabla T|^{2}(x, t) d x+\frac{\delta}{2} \int_{\Omega}|\nabla \ddot{T}|^{2}(x, t) d x .
$$

Combining (8.9)-(8.13) we obtain

$$
\begin{aligned}
G^{\prime}(t)+\epsilon H_{\delta}^{\prime}(t) \leq & -\tau_{1}\left(1-\frac{\epsilon \tau_{1}}{4}\right) \int_{\Omega}|\dddot{T}|^{2}(x, t) d x-\epsilon\left(k^{*}-\frac{\epsilon \delta}{2}\right) \int_{\Omega}|\nabla T|^{2}(x, t) d x \\
& +\epsilon\left(k \tau_{2}-\delta+\frac{2 \tau_{1}+\tau_{1}^{2}}{4 \lambda_{1}}+\frac{1}{\lambda_{1}}+\frac{k^{*}}{2}\right) \int_{\Omega}|\nabla \dot{T}|^{2}(x, t) d x \\
& +\left(k^{*} \tau_{1}+\frac{k^{*}}{2 \epsilon}+\frac{\epsilon \tau_{1}}{2 \lambda_{1}}+\frac{\delta}{2}\right) \int_{\Omega}|\nabla \ddot{T}|^{2}(x, t) d x
\end{aligned}
$$

Choosing first $\delta$ large enough such that

$$
\delta>k \tau_{2}+\frac{2 \tau_{1}+\tau_{1}^{2}}{4 \lambda_{1}}+\frac{1}{\lambda_{1}}+\frac{k^{*}}{2},
$$

and then $\epsilon$ small enough, we see that there exists a positive constant $C_{1}=C_{1}(\delta, \epsilon)$ such that

$$
\begin{aligned}
G^{\prime}(t)+\epsilon H_{\delta}^{\prime}(t) \leq & -C_{1} \int_{\Omega}\left(|\dddot{T}|^{2}+|\nabla T|^{2}+|\nabla \dot{T}|^{2}\right)(x, t) d x \\
& +\left(k^{*} \tau_{1}+\frac{k^{*}}{2 \epsilon}+\frac{\epsilon \tau_{1}}{2 \lambda_{1}}+\frac{\delta}{2}\right) \int_{\Omega}|\nabla \ddot{T}|^{2}(x, t) d x .
\end{aligned}
$$


Let us consider the Lyapunov function

$$
E(t):=F(t)+\epsilon^{2}\left(G(t)+\epsilon H_{\delta}(t)\right)
$$

which is equivalent to $F(t)$, if $\epsilon$ is small enough, i.e.,

$$
\exists d_{1}, d_{2}>0 \forall t \geq 0: d_{1} F(t) \leq E(t) \leq d_{2} F(t) .
$$

Then, by (8.4) and (8.15), we conclude

$$
\begin{aligned}
E^{\prime}(t) \leq & -C_{1} \epsilon^{2} \int_{\Omega}\left(|\dddot{T}|^{2}+|\nabla T|^{2}+|\nabla \dot{T}|^{2}\right)(x, t) d x \\
& -\left[\tau_{1}\left(k \tau_{2}-\frac{k^{*} \tau_{1}^{2}}{2}\right)-\left(\varepsilon^{2} k^{*} \tau_{1}+\frac{\varepsilon k^{*}}{2}+\frac{\epsilon^{3} \tau_{1}}{2 \lambda_{1}}+\frac{\varepsilon^{2} \delta}{2}\right)\right] \int_{\Omega}|\nabla \ddot{T}|^{2}(x, t) d x .
\end{aligned}
$$

If $\epsilon$ is chosen small enough, we get, using (8.3) and (8.16),

$$
E^{\prime}(t) \leq-c \int_{\Omega}\left(|\dddot{T}|^{2}+|\nabla T|^{2}+|\nabla \dot{T}|^{2}+|\nabla \ddot{T}|^{2}\right)(x, t) d x \leq-c_{1} E(t)
$$

for some positive constants $c, c_{1}$, implying the exponential decay of $E(t)$, and hence of $F(t)$. That is we have proved

Theorem 8.1. In the first limit case of (1.7), we have exponential energy decay, i.e.,

$$
\exists c_{1}, c_{2}>0 \forall t \geq 0: \quad F(t) \leq c_{2} \exp \left(-c_{1} t\right) F(0) .
$$

\section{References}

[1] K. Borgmeyer, Wärmeleitung und Thermoelastizität - Wohlgestelltheit von Three-PhaseLag-Modellen, Diplomarbeit (Master Thesis), University of Konstanz, 2012.

[2] D. S. Chandrasekharaiah, Hyperbolic thermoelasticity: A review of recent literature, Appl. Mech. Rev., 51(1998), 705-729.

[3] M. Dreher, R. Quintanilla and R. Racke, Ill posed problems in thermomechanics, Applied Mathematics Letters 22(2009), 1374-1379.

[4] A. E. Green and P. M. Naghdi, On undamped heat waves in an elastic solid, J. Thermal Stresses 15(1992), 253-264.

[5] A. E. Green and P. M. Naghdi, Thermoelasticity without energy dissipation, J. Elasticity, 31(1993), 189-208.

[6] R. B. Hetnarski and J. Ignaczak, Generalized thermoelasticity, J. Thermal Stresses, 22(1999), 451-470. 
[7] R. B. Hetnarski and J. Ignaczak, Nonclassical dynamical thermoelasticity, International Journal of Solids and Structures, 37(2000), 215-224.

[8] C. O. Horgan and R. Quintanilla, Spatial behaviour of solutions of the dual-phase-lag heat equation, Math. Methods Appl. Sci, 28, 43-57(2005), 43-57.

[9] J. Ignaczak, Plane Progressive Heat Waves in Metal Films , J. Thermal Stresses, 35(2012), 48-60.

[10] J. Ignaczak and M. Ostoja-Starzewski, Thermoelasticity with Finite Wave Speeds, Oxford: Oxford Mathematical Monographs, 2010.

[11] A. Miranville and R. Quintanilla, A phase-field model based on a three-phase-lag heat conduction, Applied Mathematics and Optimization, 63(2011), 133-150.

[12] S. Mukhopadhyay, S. Kothari and R. Kumar. On the representation of solutions for the theory of generalized thermoelasticity with three phase-lags. Acta Mechanica 214(2010), 305-314.

[13] R. Quintanilla, Exponential stability in the dual-phase-lag heat conduction theory, J.NonEquilibrium Thermodynamics, 27(2002), 217-227.

[14] R. Quintanilla, A condition on the delay parameters in the one-dimensional dual-phase-lag thermoelastic theory, J. Thermal Stresses, 26(2003), 713-721.

[15] R. Quintanilla and R. Racke, Qualitative aspects in dual-phase-lag thermoelasticity. SIAM Journal of Applied Mathematics. 66(2006), 9771001.

[16] R. Quintanilla and R. Racke, A note on stability of dual-phase-lag heat conduction, Int. J. Heat Mass Transfer, 49(2006), 1209-1213.

[17] R. Quintanilla and R. Racke, Qualitative aspects in dual-phase-lag heat conduction, Proc. Royal Society London A., 463(2007), 659-674.

[18] R. Quintanilla and R. Racke, A note on stability in three-phase-lag heat conduction, Int. J. Heat Mass Transfer, 51(2008), 24-29.

[19] S. K. Roy Choudhuri, On a thermoelastic three-phase-lag model, J. Thermal Stresses, 30(2007), 231-238.

[20] B. Straughan Heat Waves, Appl. Math. Sci. 177, Springer-Verlag, Berlin, 2011.

[21] D. Y. Tzou, A unified approach for heat conduction from macro- to micro-scales, ASME J. Heat Transfer, 117(1995), pp. 8-16.

[22] L. Wang, X. Zhou, and X. Wei, Heat Conduction, Mathematical Models and Analytical Solutions. Springer-Verlag, Berlin Heidelberg, 2008. 
[23] O. Weinmann, Dual-Phase-Lag Thermoelastizität. Dissertation (PhD thesis), University of Konstanz, 2009.

[24] Yang, X.: Generalized form of Hurwitz-Routh criterion and Hopf bifurcation of higher order, Appl. Math. Letters 15(2002), 615-621.

Karin Borgmeyer, Reinhard Racke

Department of Mathematics and Statistics

University of Konstanz

78457 Konstanz, Germany

karin.borgmeyer@uni-konstanz.de

reinhard.racke@uni-konstanz.de

Ramón Quintanilla

Department of Applied Mathematics 2

UPC Terrassa, Colom 11

08222 Terrassa, Spain

ramon.quintanilla@upc.edu 